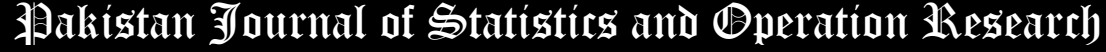

\section{Incorporation of Preferences into Supply Chains DEA Efficiency: A Geometric Attribution Approach}

\author{
Walid Abdelfattah ${ }^{1 *}$, Mohammed Sadok Cherif ${ }^{2}$
}

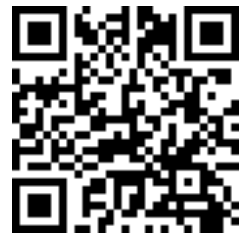

\author{
* Walid Abdelfattah \\ 1. Department of Mathematics, Northern Border University, College of Arts and Science of Rafha, Saudi Arabia, \\ walid.abdelfattah@nbu.edu.sa \\ 2. Department of Quantitative Methods, University of Monastir, Institute of Computer Science and Mathematics, \\ Tunisia, cherif_mohamed_sadok@yahoo.fr
}

\begin{abstract}
Among many applications, several studies using Data Envelopment Analysis (DEA) have examined and studied the efficiency of supply chains. However, the majority of existing approaches dealing with this research area have ignored the important factor of decision makers' preferences. The main objective of this article is to provide consistent DEA models that allow for efficiency analysis in order to determine the optimal allocation of resources according to these preferences. We propose three cases that are inspired from the geometric decomposition of preference attributions: (1) horizontal attribution, which is when decision makers treat each supply chain as a single non-detachable entity; (2) vertical attribution, which is when decision makers consider supply chains detachable and (3) combined attribution, which is when decision makers concurrently assign weights to the supply chain and to its members. Based on this suggested decomposition, new DEA models are developed, and an illustrative example is applied. The obtained results are relevant and show that DEA is capable of easily incorporating the preferences of decision-makers without resorting to weight restrictions on inputs or outputs.
\end{abstract}

Key Words: Data envelopment analysis; Efficiency; Supply chains; Decision-makers; Preferences; Geometric attribution.

\section{Mathematical Subject Classification: Operational Research}

\section{Introduction}

Within years, Data Envelopment Analysis (DEA) has become a well-known methodology to address performance measurement problems, as shown by Cook et al. 2010; Emrouznejad et al. 2008; Kaviani and Abbasi 2014; Maghbouli et al. 2014; Matin and Azizi 2015. This technique has been used in a variety of models and applications, or in more than 10,000 publications as noted by Emrouznejad and Yang (2018). Focusing on supply chains, several studies have used this technique in order to measure the corresponding efficiencies. In their review of supply chain performance measurement systems between 1998 and 2015, Balfaqih et al. (2016) found that DEA accounts for fourteen percent (a total of six) techniques adopted for their evaluation purposes.

The oldest work that addresses situations where decision-making units are interconnected, as is the case for supply chains, is that of Seiford and Zhu (1999). These authors considered a two-stage structure to measure the profitability and the tradability of 55 American commercial banks. They used a DEA approach that ignored the potential conflicts between the first and second stages arising from intermediate variables. By studying the efficiency measurement of 24 non-life insurance companies in Taiwan, Kao and Hwang (2008) decomposed efficiency into a two-stage process in which each stage represents a sub-production process. The authors have developed a modified DEA standard model where they proposed that the overall efficiency is the product of the two stages' efficiencies. Chen et al. (2009) have 
combined the efficiency of a whole two-stage process as the mean weighted efficiency of the two separate stages. Wang and Chin (2010) have generalized the obtained combined model by introducing relative weights for the two separate stages. These works have used the DEA for measuring only the efficiency of multi-stage processes. However, this does not deny that the proposed models could be applied to supply chains problems. Indeed, a supply chain is a multi-level (stage) process. In this regard, Liang et al. (2006) and Yang et al. (2009) have reported that in the context of DEA, there are a number of methods that have the potential to evaluate supply chains' efficiency. Tavana et al. (2016) have asserted that two-stage DEA models could be used to analyze the inner relationships among the components of a supply chain, but the models must be designed in a suitable manner. Indeed, production processes comprise subunits that are connected to each other through a network. The output of a subunit may be the input of another subunit, and these interactions ultimately result in the final output production.

Many studies have directly addressed the efficiency of supply chains using DEA. Zhu (2003) proposed a model allowing for the definition and measurement of the overall efficiency of a dyadic supply chain (a two-member supply chain) composed of a seller and a buyer as well as those of its members. This author presented a set of optimal values for the intermediate variables that establish an efficient supply chain. Using the same considered chain, Liang et al. (2006) developed two DEA models in order to measure the supply chain efficiency when intermediate variables are incorporated into the evaluation procedure. In the first model, the buyer-seller relationship is modeled as a two-stage non-cooperative game, and in the second model, the buyer and the seller operate in a cooperative context. Chen et al. (2006) analyzed the relations between the efficiencies of supply chain members using a model called the Supply chainDEA Game model. By considering a supplier-producer supply chain, the authors proposed several supply chain efficiency functions designed to identify the inefficiency by developing two efficiency functions for the producer and for the supplier. They have shown that these two functions are inverses. Recently, Tavana et al. (2016) proposed a two-stage DEA model. The purpose of their model is to evaluate an entire three-level supply chain comprised of a supplier, manufacturer and distributor. Abdelfattah and Rebai (2016) considered a supplier-producer supply chain with intermediate measures. They developed different DEA models based on the differentiation between eight different situations by distinguishing between the cooperation concept and the dominance concept. More recently, Zhai et al. (2019) developed a DEA model based on frontier-shift-methodology to measure the ecological efficiency of energy supply chains in 16 cities in one of the Chinese provinces. By considering a two-stage process, these authors showed that efficiency could be derived only and only if both stages obtain the optimal solutions. Arabshahi et al. (2020) proposed a DEA-based model to evaluate supply chain performance in order to identify efficient members. By adopting a seller-buyer structure, the authors assumed the existence of only one decision maker who controls the whole supply chain.

If the type of relationship between members constituting supply chains was considered in most studies, it was at the expense of taking into account decision makers (DMs) who supervise and control the chains and who have an important role in supply chains' operations. Indeed, we find that the literature has not frequently addressed this subject when it is associated with the incorporation of decision makers' preferences (DMsP). This deficiency can be explained by several reasons. The most important reason is that in DEA models, input and output weights are considered as integrated decision variables, and no additional information is needed to reach the reference point. Consequently, these models in their standard form can generate incoherent solutions with the operational DMsP. Therefore, some scientific research has been conducted to set the constraints to the weights, and thus, DMsP can be incorporated into the assessment of efficiency. In this same context, Allen et al. (1997) presented a review on the evolution of the use of weight restrictions and value judgements in DEA. Cook and Seiford (2009) classified DEA models involving weight restrictions into five categories. Saen (2010) proposed a weight restriction DEA model for the supplier selection problem with the consideration of dual-role factors. Contreras (2011) offered a procedure consisting of two stages. First, a DEA-inspired model for the aggregation of preferences is applied. Second, in order to obtain a group solution, the procedure derives a compromise solution by determining a social vector of weights for evaluating the complete set of alternatives.

Instead of weight restrictions, there are authors who have used an additional multi-criteria decision making technique besides DEA in order to incorporate DMsP into the evaluation process. Wang et al. (2018) used first Fuzzy Analytic Network Process to evaluating and ranking the considered criteria, DEA is then proposed in order to select the efficient suppliers. Alcaide-López-de-Pablo et al. (2014) evoked works addressing this issue, where efficiency scores obtained by the DEA are generally used in the objective function of the associated mathematical programming problems, or conversely, efficiency scores are computed after solving the corresponding mathematical programming problems. Among others, Zhao and Sun (2008) proposed a preference restraint DEA approach for supplier selection in which 
the DMsP on inputs and outputs are given by the Analytic Hierarchy Process method. Parkan and Wang (2007) applied DEA and the Operational Competitiveness Rating Analysis to evaluate a global electronic component distributor. Xu et al. (2009) used rough-set theory and DEA techniques in a furniture manufacturing supply chain in China. Wong (2009) combined DEA with Monte Carlo simulation to evaluate supply chain performance in a stochastic environment.

In this paper, the authors propose a novel approach that allows for and maintains the inherent philosophy of the DEA methodology. In addition, it incorporates of the DMsP into DEA models when assessing supply chains' efficiency without modifying the weights of the inputs and outputs that are assumed as variables in these models, and it does not use any other methods. Moreover, this work proposes that DMsP should be incorporated and not aggregated into DEA models. In other words, they should appear with the input and output weights. In fact, in the same manner that DMs have preferences on inputs and outputs, they could also have judgements on supply chains and the relative members themselves.

The organization of the paper is as follows. In the next section, we introduce the proposed approach to incorporate DMsP into the efficiency measurement procedure. After that, new DEA models are developed based on what was proposed in the preceding section. An illustrative example is applied in which we compare the efficiencies that are yielded in different manners of preferences attribution, and a discussion is conducted on the basis of the found results. Finally, conclusions are drawn.

\section{Incorporation of decision makers' preferences DMsP}

This works aims to provide DEA models for measuring supply chains' efficiency while taking into consideration DMsP. Herein, first, the weights representing these preferences (beside input and output weights) are established without having to resort to weight restrictions, as is often done in the literature. Second, the model relies only on the DEA method. Therefore, no additional alternatives or methods are used. To achieve these goals, the supply chains considered here are dyadic supply chains with independent inputs (Figure1), which are defined as linear minimal chains composed only by two units (firms, members). In fact, given its simplicity, authors often advise using it first for the performance evaluation and then to iterate the reasoning to more complex chains. Moreover, this work assumes the following assumptions.

1. Several DMs control and manage the considered supply chains.

2. DMs can express their judgments and interactivity by attributing weights to each member and/or to the totality of each supply chain.

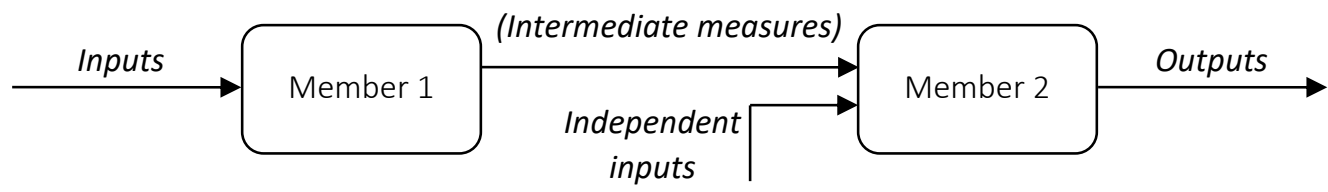

Figure 1: Dyadic supply chain with independent inputs.

The closest work to consider DMs when measuring supply chains' efficiencies is that of Chen et al. (2006). These authors separated two cases of supply chain controls: (1) the Decentralized Control System where the first and the second members control their own efficiencies and (2) the Central Control System where a single DM, who is different from the members that constitute the chain, monitors the supply chain. In this paper, the case of decentralized control corresponds to the case where DMs, if they are even present, do not affect the procedure of measuring the supply chain's efficiency. In other words, DMs are supposed to be indifferent and neutral about all chains and the members constituting these chains. Therefore, this situation assumes the absence of preferences. On the other hand, for centralized control, the authors propose treating it differently by using a Geometric Attribution Approach (GAA) of DMsP. DMs could allocate their preferences' weights to chains and their constituent members in a horizontal and/or in a vertical manner. 
The GAA is especially inspiring because a given DM may treat each supply chain as a single non-detachable entity, and as a result, he could assign a relative weight to each supply chain independent of its members. This case corresponds to a horizontal attribution of preferences. Then, when that DM considers the supply chains detachable, he will assign a relative weight to the first member of each chain and another one to its second member. This case corresponds to a vertical attribution of preferences. Meanwhile, another case could be generated that combines these two situations where a DM concurrently assigns weights to the supply chain and to its members. This case will correspond to a combined attribution of preferences. Figure 2 summarizes all these ways considered by the GAA.

The attribution of preferences following these proposed cases is not a goal in itself, but the aim is rather to assess supply chains' efficiency while considering DMsP. Therefore, three DEA models will be introduced, and each model treats a case among the three cases proposed for DMsP attribution. In the third situation of combined attribution, we develop a general DEA model that allows the grouping of all these situations.

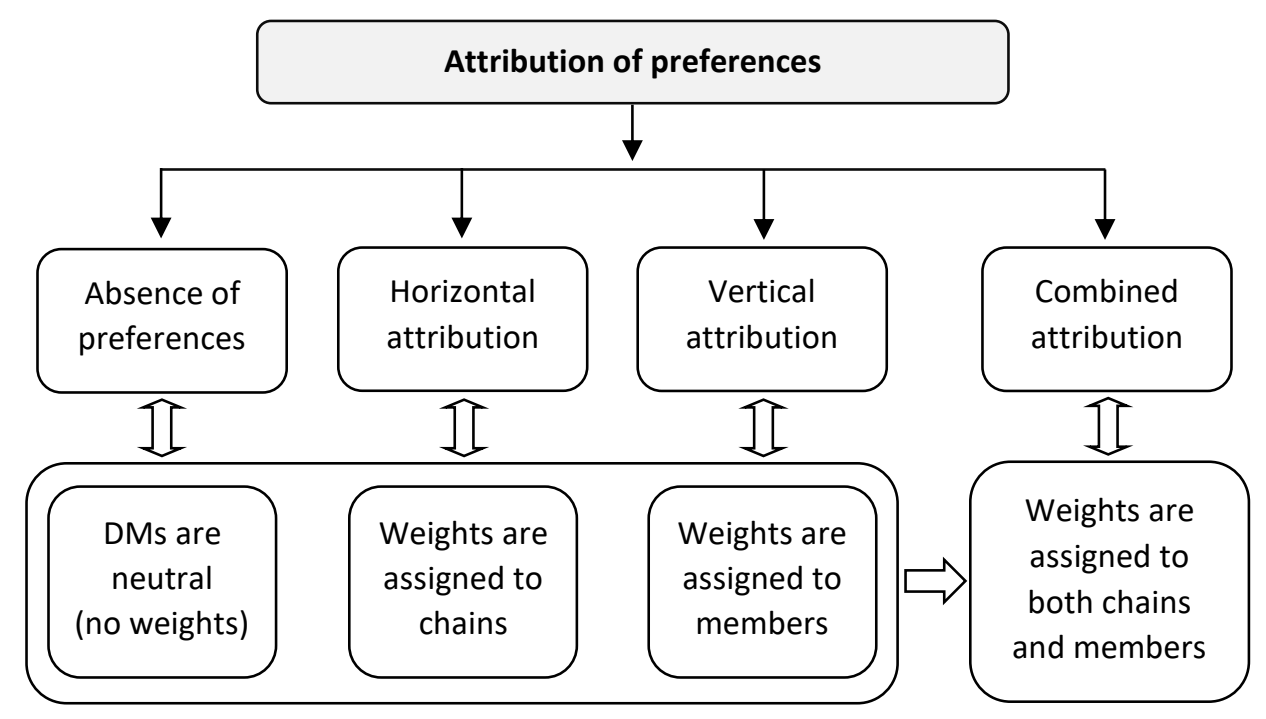

Figure 2: The geometric attribution approach of decision makers' preferences.

\section{The proposed models}

While taking into account suggestions when considering DMsP attribution, the DEA modeling will be based on one of the different situations proposed by Abdelfatta and Rebai (2016). These authors have suggested that members of a given supply chain could operate in one of two different contexts of cooperation (cooperative or non-cooperative). For each context, there could be four different situations of dominance (Absence of dominance, dominance of the first member, dominance of the second member and egalitarian dominance). In this article, the DEA model, which represents the situation where DMs are supposed to be neutral, will be the same as the model given by these authors in the situation where members constituting the supply chain are supposed to be cooperative without any kind of dominance.

Let us consider a set of $Z$ dyadic supply chains $(j=1, \ldots, Z) \cdot x_{i S_{j}}$ is the quantity of input $i(i=1, \ldots, I)$ consumed by the first member $S_{j}, v_{i}$ is the weight related to the input $i, x_{p M_{j}}$ is the quantity of independent input $p(p=1, \ldots, P)$ consumed by the second member $M_{j}, v_{p}$ is the weight related to the input $p, y_{q M_{j}}$ represents the output $q(q=1, \ldots, Q)$ produced by the second member $M_{j}, \mu_{q}$ is the weight related to the output $q, w_{r S C_{j}}$ is the quantity of intermediate measures $r(r=1, \ldots, R)$ produced by the first member $S_{j}$ and consumed by the second member $M_{j}, S_{j}$ and $M_{j}$ together compose the chain $S C_{j}$, and $t_{r}$ is the weight related to the intermediate measures $r$.

Then, for a given $k^{\text {th }}$ dyadic supply chain, the case of absence of preferences is given by the following model: 


$$
\begin{aligned}
& \operatorname{Max} \frac{1}{2}\left(\sum_{q=1}^{Q} \mu_{q} y_{q M_{k}}+\sum_{r=1}^{R} t_{r}^{S} w_{r S C_{k}}\right) \\
& \text { Subject to } \\
& \quad \sum_{i=1}^{I} \vartheta_{i} x_{i S_{k}}=1 ; \\
& \quad \sum_{r=1}^{R} t_{r}^{S} w_{r S C_{j}}-\sum_{i=1}^{I} \vartheta_{i} x_{i S_{j}} \leq 0 ; j=1, \ldots, Z ; \\
& \sum_{r=1}^{R} t_{r}^{M} w_{r S C_{k}}+\sum_{p=1}^{P} \vartheta_{p} x_{p M_{k}}=1 ; \\
& \quad \sum_{q=1}^{Q} \mu_{q} y_{q M_{j}}-\sum_{r=1}^{R} t_{r}^{M} w_{r S C_{j}}-\sum_{p=1}^{P} \vartheta_{p} x_{p M_{j}} \leq 0 ; j=1, \ldots, Z ; \\
& t_{r}^{S}, t_{r}^{M}, \vartheta_{i}, \mu_{q}, \vartheta_{p} \geq 0 ; r=1, \ldots, R ; i=1, \ldots, I ; p=1, \ldots, P ; q=1, \ldots, Q .
\end{aligned}
$$

This model retains only the weights that represent inputs and outputs and discards any kind of weights that represent preferences. The objective function considers the mean between the first member's output (intermediate measures) and the second member's output. Therefore, apart from the weighted sum of the first member's inputs, the weighted sum of the second member's inputs should be equal to one.

Based on this model, new DEA models will be developed. Therefore, three different situations will be retained: the horizontal attribution of preferences, the vertical attribution of preferences and the combined model.

\section{Horizontal attribution of preferences}

By adopting a horizontal attribution of preferences, DMs treat each chain as a non-detachable entity. Let us assume the existence of $D$ DMs $(d=1, \ldots, D)$ who subjectively reveal weights that reflect their preferences toward each of these chains. We denote $\alpha_{d j}$ as the weight assigned by DM $d$ to the chain $j(j=1, \ldots, Z)$, and $E_{S C_{k}}^{H}$ as the efficiency of the $k^{t h}$ chain. Model (2) gives this efficiency.

$$
\operatorname{Max} \frac{1}{2} \frac{1}{D} \sum_{d=1}^{D} \alpha_{d k}\left(\sum_{q=1}^{Q} \mu_{q} y_{q M_{k}}+\sum_{r=1}^{R} t_{r}^{S} w_{r S C_{k}}\right)
$$

Subject to

$$
\begin{aligned}
& \sum_{i=1}^{I} \vartheta_{i} x_{i S_{k}}=1 ; \\
& \frac{1}{D} \sum_{d=1}^{D} \alpha_{d j} \sum_{r=1}^{R} t_{r}^{S} w_{r S C_{j}}-\sum_{i=1}^{I} \vartheta_{i} x_{i S_{j}} \leq 0 ; j=1, \ldots, Z ; \\
& \sum_{r=1}^{R} t_{r}^{M} w_{r S C_{k}}+\sum_{p=1}^{P} \vartheta_{p} x_{p M_{k}}=1 ; \\
& \frac{1}{D} \sum_{d=1}^{D} \alpha_{d j} \sum_{q=1}^{Q} \mu_{q} y_{q M_{j}}-\sum_{r=1}^{R} t_{r}^{M} w_{r S C_{j}}-\sum_{p=1}^{P} \vartheta_{p} x_{p M_{j}} \leq 0 ; j=1, \ldots, Z ; \\
& \sum_{j=1}^{Z} \alpha_{d j}=1 ; \\
& 0 \leq \alpha_{d j} \leq 1 ; \\
& t_{r}^{S}, t_{r}^{M}, \vartheta_{i}, \mu_{q}, \vartheta_{p} \geq 0 ; r=1, \ldots, R ; i=1, \ldots, I ; p=1, \ldots, P ; q=1, \ldots, Q .
\end{aligned}
$$


The weighted sum of DMsP $\left(\frac{1}{D} \sum_{d=1}^{D} \alpha_{d k}\right)$ is incorporated into the objective function. Given that the model that is adopted herein is output-oriented, this expression is multiplied only by the weighted sums of outputs. Note that preferences are considered subjectively, so it is first necessary to set $\sum_{j=1}^{Z} \alpha_{d j}=1$ and $0 \leq \alpha_{d j} \leq 1$.

\section{Vertical attribution of preferences}

This way of attributing preferences consists of supposing that detachable chains constitute the sample under study. That is, each DM $d(d=1, \ldots, D)$ assigns a weight $\beta_{d S_{k}}$ to the first member $S_{k}$ and another weight $\beta_{d M_{k}}$ to the second member $M_{k}$. Let us denote $E_{S C_{k}}^{V}$ as the efficiency of the $k^{\text {th }}$ chain in the case of the vertical attribution of preferences. Model (3) gives this efficiency.

$$
\begin{aligned}
& \text { Max } \frac{1}{2} \frac{1}{D}\left(\sum_{d=1}^{D} \beta_{d M_{k}} \sum_{q=1}^{Q} \mu_{q} y_{q M_{k}}+\sum_{d=1}^{D} \beta_{d s_{k}} \sum_{r=1}^{R} t_{r}^{S} w_{r S C_{k}}\right) \\
& \text { Subject to } \\
& \quad \sum_{i=1}^{I} \vartheta_{i} x_{i S_{k}}=1 ; \\
& \quad \frac{1}{D} \sum_{d=1}^{D} \beta_{d S_{j}} \sum_{r=1}^{R} t_{r}^{S} w_{r S C_{j}}-\sum_{i=1}^{I} \vartheta_{i} x_{i S_{j}} \leq 0 ; j=1, \ldots, Z ; \\
& \sum_{r=1}^{R} t_{r}^{M} w_{r S C_{k}}+\sum_{p=1}^{P} \vartheta_{p} x_{p M_{k}}=1 ; \\
& \quad \frac{1}{D} \sum_{d=1}^{D} \beta_{d M_{j}} \sum_{q=1}^{Q} t_{q} y_{q M_{j}}-\sum_{r=1}^{R} t_{r}^{M} w_{r S C_{j}}-\sum_{p=1}^{P} \vartheta_{p} x_{p M_{j}} \leq 0 ; j=1, \ldots, Z ; \\
& \sum_{j=1}^{Z} \beta_{d S_{j}}=1 ; \\
& \sum_{Z}^{Z} \beta_{d M_{j}}=1 ; \\
& \sum_{j=1} \leq \beta_{d M_{j}} \leq 1 ; ; \\
& t_{r}^{S}, t_{r}^{M}, \vartheta_{i}, \mu_{q}, \vartheta_{p} \geq 0 ; r=1, \ldots, R ; i=1, \ldots, I ; p=1, \ldots, P ; q=1, \ldots, Q .
\end{aligned}
$$

In this model, the weighted sum of DMsP regarding the first member is multiplied by the weighted sum of the latter's outputs (also the intermediate measures). Meanwhile, the preference weights regarding the second member are multiplied by the weighted sum of this member outputs. Recall that preferences are considered subjectively, so it is first necessary to set $\sum_{j=1}^{Z} \beta_{d S_{j}}=1, \sum_{j=1}^{Z} \beta_{d M_{j}}=1,0 \leq \beta_{d S_{j}} \leq 1$ and $0 \leq \beta_{d M_{j}} \leq 1$.

\section{Combined model}

This case combines the two previous models into only one model in a way such that DMs are totally flexible, whether they treat each supply chain as a single non-detachable entity (horizontal attribution of preferences) and/or they consider it detachable (vertical attribution of preferences). The first model, which corresponds to situation of neutrality of DMs, is systematically considered.

In order to achieve the aggregation of all previous situations into one model, this work proposes that each chain $k, f_{k}\left(a_{k}, b_{k}\right)$ be a joint function of $a_{k}$ and $b_{k}$, which is defined as follows:

$$
f_{k}\left(a_{k}, b_{k}\right)=\left\{\begin{array}{rr}
0 & \text { if } a_{k}=0 \text { and } b_{k}=0 \\
b_{k} & \text { if } a_{k}=0 \\
a_{k} & \text { if } b_{k}=0 \\
\frac{a_{k}+b_{k}}{2} & \text { if } a_{k} \neq 0 \text { and } b_{k} \neq 0
\end{array}\right.
$$


Where $a_{k}=\frac{1}{D} \sum_{d=1}^{D} \alpha_{d k}$ and $b_{k}=\frac{1}{D} \sum_{d=1}^{D} \beta_{d k}$

$\alpha_{d k}$ is the preference weight assigned by a DM $d$ to a given chain $k$, and $\beta_{d k}$ is the preference weight assigned by a DM $d$ to one of the two members. This establishes a given chain $k$ so that $\beta_{d k}=\beta_{d s_{k}}$ when it is the first member, and $\beta_{d k}=\beta_{d M_{k}}$ when it is the second member. At this point, we make this a joint function written as only one expression instead of four by eliminating all the conditions on it. For this purpose, the ceiling function will be used.

Recall that the ceiling function (also known as the least integer function) of a real number $x$, which is denoted as $\lceil x\rceil$, is defined as the smallest integer that is greater than or equal to $x$. In general, $\lceil x\rceil$ is the unique integer satisfying $\lceil x\rceil-$ $1<x \leq\lceil x\rceil$. By taking $x \in[0,1]$, $\lceil x\rceil$ will have the following values:

$$
\lceil x\rceil= \begin{cases}0 & \text { if } x=0 \\ 1 & \text { if } x \neq 0\end{cases}
$$

For a given member of the chain $k$, the proposed function that aggregates the weights denoted by $P_{k}$ is given by the following expression:

$$
\frac{\left[\left(a_{k}+1\right)^{\left[a_{k}\right]}-1\right]+\left[\left(b_{k}+1\right)^{\left\lceil b_{k}\right]}-1\right]}{2^{\left[a_{k}\right]+\left[b_{k}\right\rceil-1}}
$$

We can easily verify that $P_{k}\left(a_{k}, b_{k}\right)=f_{k}\left(a_{k}, b_{k}\right)$

As illustration of this proposed function, we assume the existence of four DMs whose weights assigned to ten considered chains are summarized in Table 1, while the weights assigned to the chains' members are shown in Table 2. The obtained values of the function $P_{k}\left(a_{k}, b_{k}\right)$ are given in Table 3.

\begin{tabular}{|c|c|c|c|c|c|}
\hline \multirow{2}{*}{$\begin{array}{l}\text { Chain } \\
\mathrm{n}^{\circ}: k\end{array}$} & \multicolumn{4}{|c|}{$\alpha_{d k}$} & \multirow{2}{*}{ Mean } \\
\hline & $\alpha_{1 k}$ & $\alpha_{2 k}$ & $\alpha_{3 k}$ & $\alpha_{4 k}$ & \\
\hline 1 & 0.12 & 0.09 & 0.05 & 0.18 & 0.1100 \\
\hline 2 & 0.08 & 0.10 & 0.15 & 0.12 & 0.1125 \\
\hline 3 & 0.09 & 0.06 & 0.14 & 0.00 & 0.0725 \\
\hline 4 & 0.14 & 0.11 & 0.10 & 0.00 & 0.0875 \\
\hline 5 & 0.08 & 0.17 & 0.09 & 0.10 & 0.1100 \\
\hline 6 & 0.11 & 0.12 & 0.12 & 0.06 & 0.1025 \\
\hline 7 & 0.13 & 0.00 & 0.11 & 0.09 & 0.0825 \\
\hline 8 & 0.06 & 0.09 & 0.11 & 0.15 & 0.1025 \\
\hline 9 & 0.10 & 0.11 & 0.08 & 0.13 & 0.1050 \\
\hline 10 & 0.09 & 0.15 & 0.05 & 0.17 & 0.1150 \\
\hline
\end{tabular}

Table 1: weights assigned by DMs to chains 
Table 2: weights assigned by DMs to chains' members

\begin{tabular}{|c|c|c|c|c|c|c|c|c|c|c|}
\hline \multirow{2}{*}{$\begin{array}{l}\text { Chain } \\
\mathrm{n}^{\circ}: k\end{array}$} & \multicolumn{4}{|c|}{$\beta_{d s_{k}}$} & \multicolumn{4}{|c|}{$\beta_{d M_{k}}$} & \multicolumn{2}{|c|}{ Mean } \\
\hline & $\beta_{1 S_{k}}$ & $\beta_{2 S_{k}}$ & $\beta_{3 S_{k}}$ & $\beta_{4 S_{k}}$ & $\beta_{1 M_{k}}$ & $\beta_{2 M_{k}}$ & $\beta_{3 M_{k}}$ & $\beta_{4 M_{k}}$ & $\beta_{d S_{k}}$ & $\beta_{d M_{k}}$ \\
\hline 1 & 0.14 & 0.07 & 0.10 & 0.09 & 0.09 & 0.12 & 0.14 & 0.06 & 0.1000 & 0.1025 \\
\hline 2 & 0.09 & 0.12 & 0.06 & 0.11 & 0.12 & 0.09 & 0.09 & 0.11 & 0.0950 & 0.1025 \\
\hline 3 & 0.10 & 0.09 & 0.13 & 0.00 & 0.08 & 0.16 & 0.13 & 0.00 & 0.0800 & 0.0925 \\
\hline 4 & 0.12 & 0.05 & 0.12 & 0.10 & 0.11 & 0.13 & 0.10 & 0.09 & 0.0975 & 0.1075 \\
\hline 5 & 0.08 & 0.15 & 0.00 & 0.12 & 0.14 & 0.08 & 0.00 & 0.14 & 0.0875 & 0.0900 \\
\hline 6 & 0.11 & 0.12 & 0.09 & 0.07 & 0.12 & 0.07 & 0.11 & 0.10 & 0.0975 & 0.1000 \\
\hline 7 & 0.06 & 0.13 & 0.16 & 0.00 & 0.08 & 0.10 & 0.07 & 0.08 & 0.0875 & 0.0825 \\
\hline 8 & 0.12 & 0.08 & 0.08 & 0.16 & 0.10 & 0.12 & 0.10 & 0.13 & 0.1100 & 0.1125 \\
\hline 9 & 0.08 & 0.10 & 0.13 & 0.20 & 0.10 & 0.06 & 0.11 & 0.20 & 0.1275 & 0.1175 \\
\hline 10 & 0.10 & 0.09 & 0.13 & 0.15 & 0.06 & 0.07 & 0.15 & 0.09 & 0.1175 & 0.0925 \\
\hline
\end{tabular}

Table 3: Values of the weight aggregation function $\mathrm{P}_{\mathrm{k}}\left(\mathrm{a}_{\mathrm{k}}, \mathrm{b}_{\mathrm{k}}\right)$

\begin{tabular}{lccccc}
\hline $\begin{array}{l}\text { Chain } \\
\mathrm{n}^{\circ}: k\end{array}$ & $a_{k}$ & $b_{s k}$ & $b_{M k}$ & $P_{k}\left(a_{k}, b_{s k}\right)$ & $P_{k}\left(a_{k}, b_{M k}\right)$ \\
\hline 1 & 0.110 & 0.100 & 0.103 & 0.105 & 0.106 \\
2 & 0.113 & 0.095 & 0.103 & 0.104 & 0.108 \\
3 & 0.073 & 0.080 & 0.093 & 0.076 & 0.083 \\
4 & 0.088 & 0.098 & 0.108 & 0.093 & 0.098 \\
5 & 0.110 & 0.088 & 0.090 & 0.099 & 0.100 \\
6 & 0.103 & 0.098 & 0.100 & 0.100 & 0.101 \\
7 & 0.083 & 0.088 & 0.083 & 0.085 & 0.083 \\
8 & 0.103 & 0.110 & 0.113 & 0.106 & 0.108 \\
9 & 0.105 & 0.128 & 0.118 & 0.116 & 0.111 \\
10 & 0.115 & 0.118 & 0.093 & 0.116 & 0.104 \\
\hline
\end{tabular}

Let us denote $E_{S C_{k}}^{C}$ as the efficiency of the $k^{\text {th }}$ chain in the case of the combined attribution of preferences. Model (7) gives this efficiency.

$$
\operatorname{Max} \frac{1}{2}\left[P_{k}\left(a_{k}, \beta_{d M_{k}}\right) \sum_{q=1}^{Q} \mu_{q} y_{q M_{k}}+P_{k}\left(a_{k}, b_{s k}\right) \sum_{r=1}^{R} t_{r}^{S} w_{r S C_{k}}\right]
$$

Subject to

$$
\begin{aligned}
& \sum_{i=1}^{I} \vartheta_{i} x_{i S_{k}}=1 ; \\
& P_{k}\left(a_{j}, b_{s j}\right) \sum_{r=1}^{R} t_{r}^{S} w_{r S C_{j}}-\sum_{i=1}^{I} \vartheta_{i} x_{i S_{j}} \leq 0 ; j=1, \ldots, Z ; \\
& \sum_{r=1}^{R} t_{r}^{M} w_{r S C_{k}}+\sum_{p=1}^{P} \vartheta_{p} x_{p M_{k}}=1 ;
\end{aligned}
$$




$$
\begin{aligned}
& P_{k}\left(a_{j}, b_{M j}\right) \sum_{q=1}^{Q} \mu_{q} y_{q M_{j}}-\sum_{r=1}^{R} t_{r}^{M} w_{r S C_{j}}-\sum_{p=1}^{P} \vartheta_{p} x_{p M_{j}} \leq 0 ; j=1, \ldots, Z ; \\
& t_{r}^{S}, t_{r}^{M}, \vartheta_{i}, \mu_{q}, \vartheta_{p} \geq 0 ; r=1, \ldots, R ; i=1, \ldots, I ; p=1, \ldots, P ; q=1, \ldots, Q .
\end{aligned}
$$

Based on the proposed weight aggregation function $P_{k}\left(\alpha_{d k}, \beta_{d k}\right)$, this model naturally yields a zero efficiency for a given supply chain $k$ when all DMs decide to set $\alpha_{d k}, \beta_{d s_{k}}$ and $\beta_{d M_{k}}$ all equal to zero. Thus, when a given chain shows a zero efficiency, it means that it is out of consideration. Moreover, when a DM decides to assign a preference weight to a whole given chain $\alpha_{d k} \neq 0$ with another preference weight regarding one of the members as $\beta_{d k} \neq 0$, this model will consider the average between the two weights.

\section{Illustrative example}

The illustration considered here assumes the existence of ten dyadic supply chains, three inputs consumed by the first member, three intermediate measures, one independent input consumed by the second member and two outputs produced by the same member (Table 4). It also supposes the existence of four DMs who subjectively attribute weights reflecting their preferences toward the chains and toward the members constituting these chains.

Thus, the weights assigned by DMs to chains (Table 1) are used in order to calculate the efficiency scores in the situation of the horizontal attribution of preferences. Meanwhile, the weights assigned by DMs to members (Table 2) are used to determine those scores in the situation of the vertical attribution of preferences. Finally, in the situation of combined attribution, the aggregated weights given by the function $P_{k}\left(a_{k}, b_{k}\right)$ in Table 3 are used.

For a given chain $k$, the first member efficiency in the case of the absence of preferences $\left(E_{S_{k}}\right)$ can be obtained by considering the weighted sum of its outputs $\sum_{r=1}^{R} t_{r}^{S^{*}} w_{r S C_{k}}$, where $t_{r}^{S^{*}}$ are the optimal weights assigned to the intermediate measures $r$ that act as output. Thus, in the cases of horizontal, vertical or combined attributions of DMsP, this efficiency $\left(E_{S_{k}}^{H}, E_{S_{k}}^{V}\right.$ or $\left.E_{S_{k}}^{C}\right)$ is obtained by multiplying the previous weighted sum by the corresponding weighted sum of DMsP, which is $a_{k}, b_{s k}$ or $P_{k}\left(\alpha_{d k}, \beta_{d s_{k}}\right)$, respectively.

With the same manner, the second member efficiency in the case of the absence of preferences $\left(E_{M_{k}}\right)$ is given by calculating the weighted sum of its outputs $\sum_{q=1}^{Q} \mu_{q}^{*} y_{q M_{k}}$, where $\mu_{q}^{*}$ are the optimal weights assigned to the output $q$ of the second member. Meanwhile, in the cases of horizontal, vertical or combined attributions of DMs' preferences, the second member efficiency $\left(E_{M_{k}}^{H}, E_{M_{k}}^{V}\right.$ or $\left.E_{M_{k}}^{C}\right)$ is obtained by multiplying this sum by the corresponding weighted sum of DMs' preferences, which is $a_{k}, b_{M k}$ or $P_{k}\left(a_{k}, b_{k}\right)$, respectively.

The total chain efficiency $\left(E_{S C_{k}}, E_{S C_{k}}^{H}, E_{S C_{k}}^{V}\right.$ or $E_{S C_{k}}^{C}$ ) can be determined directly from the resolution of the relative model or by deducing the average between its constituting members' efficiencies. Thus, a given chain could display an efficiency score equal to one if and only if its two members are both efficient. 
Table 4: Data of ten dyadic supply chains

\begin{tabular}{|c|c|c|c|c|c|c|c|c|c|}
\hline \multirow{2}{*}{$\begin{array}{l}\text { Chain } \\
n^{\circ}: k\end{array}$} & \multicolumn{3}{|c|}{$X_{S_{k}}$} & \multicolumn{3}{|c|}{$W_{S C_{k}}$} & \multirow{2}{*}{$\begin{array}{l}X_{M_{k}} \\
x_{1 M_{k}}\end{array}$} & \multicolumn{2}{|c|}{$Y_{M_{k}}$} \\
\hline & $x_{1 s_{k}}$ & $x_{2 s_{k}}$ & $x_{3 s_{k}}$ & $w_{1 S C_{k}}$ & $w_{2 S C_{k}}$ & $w_{3 S C_{k}}$ & & $y_{1 M_{k}}$ & $y_{2 M_{k}}$ \\
\hline 1 & 9 & 50 & 1 & 20 & 10 & 5 & 8 & 100 & 25 \\
\hline 2 & 10 & 18 & 10 & 10 & 15 & 7 & 10 & 70 & 20 \\
\hline 3 & 9 & 30 & 3 & 8 & 20 & 2 & 8 & 96 & 30 \\
\hline 4 & 8 & 25 & 1 & 20 & 20 & 10 & 10 & 80 & 20 \\
\hline 5 & 10 & 40 & 5 & 25 & 20 & 5 & 15 & 85 & 15 \\
\hline 6 & 7 & 35 & 2 & 35 & 10 & 5 & 5 & 90 & 35 \\
\hline 7 & 7 & 30 & 3 & 10 & 25 & 8 & 10 & 100 & 30 \\
\hline 8 & 12 & 40 & 4 & 20 & 25 & 4 & 8 & 120 & 10 \\
\hline 9 & 9 & 25 & 2 & 10 & 10 & 5 & 15 & 110 & 15 \\
\hline 10 & 10 & 50 & 1 & 20 & 15 & 9 & 10 & 80 & 20 \\
\hline
\end{tabular}

Obtained results are summarized in Table 5. It is remarkable that only chain 6 has retained its position as a reference chain in all the situations of the preference allocations. This is because each member constituting this chain has retained an efficiency score equal to one. On the other side, the lowest level of efficiency (0.576) was recorded by chain 5 in the case of the vertical allocation of preferences.

The solution differs from a member to another and from a chain to another. There are chains and members that have experienced either an improvement or a degradation in their efficiency according to the different considered cases. Moreover, Figure 3 shows this variation in chains' efficiencies for each of these cases.

Table 5: Chains and members' efficiencies yielded in different ways of preference attributions

\begin{tabular}{|c|c|c|c|c|c|c|c|c|c|c|c|c|}
\hline \multirow{2}{*}{$\begin{array}{l}\text { Chain } \\
\mathrm{n}^{\circ}: \mathrm{k}\end{array}$} & \multicolumn{3}{|c|}{ No preferences } & \multicolumn{3}{|c|}{ Horizontal att. } & \multicolumn{3}{|c|}{ Vertical att. } & \multicolumn{3}{|c|}{ Combined att. } \\
\hline & $\mathrm{E}_{\mathrm{S}_{\mathrm{k}}}$ & $\mathrm{E}_{\mathrm{M}_{\mathrm{k}}}$ & $\mathrm{E}_{\mathrm{SC}_{\mathrm{k}}}$ & $\mathrm{E}_{S_{\mathrm{k}}}^{\mathrm{H}}$ & $\mathrm{E}_{\mathrm{M}_{\mathrm{k}}}^{\mathrm{H}}$ & $\mathrm{E}_{\mathrm{S} \mathrm{C}_{\mathrm{k}}}^{\mathrm{H}}$ & $\mathrm{E}_{\mathrm{S}_{\mathrm{k}}}^{\mathrm{V}}$ & $\mathrm{E}_{\mathrm{M}_{\mathrm{k}}}^{\mathrm{V}}$ & $\mathrm{E}_{\mathrm{SC}_{\mathrm{k}}}^{\mathrm{V}}$ & $E_{S_{k}}^{C}$ & $\mathrm{E}_{\mathrm{M}_{\mathrm{k}}}^{\mathrm{C}}$ & $\mathrm{E}_{\mathrm{SC}_{\mathrm{k}}}^{\mathrm{C}}$ \\
\hline 1 & 1.000 & 1.000 & 1.000 & 0.990 & 1.000 & 0.995 & 0.889 & 1.000 & 0.944 & 0.937 & 1.000 & 0.969 \\
\hline 2 & 1.000 & 0.805 & 0.903 & 1.000 & 1.000 & 1.000 & 1.000 & 0.863 & 0.932 & 1.000 & 0.972 & 0.986 \\
\hline 3 & 0.800 & 1.000 & 0.900 & 0.680 & 1.000 & 0.840 & 0.694 & 1.000 & 0.847 & 0.544 & 1.000 & 0.772 \\
\hline 4 & 1.000 & 0.628 & 0.814 & 1.000 & 0.575 & 0.788 & 1.000 & 0.675 & 0.838 & 1.000 & 0.630 & 0.815 \\
\hline 5 & 0.676 & 0.604 & 0.640 & 0.862 & 0.715 & 0.788 & 0.644 & 0.509 & 0.576 & 0.865 & 0.673 & 0.769 \\
\hline 6 & 1.000 & 1.000 & 1.000 & 1.000 & 1.000 & 1.000 & 1.000 & 1.000 & 1.000 & 1.000 & 1.000 & 1.000 \\
\hline 7 & 1.000 & 0.833 & 0.917 & 1.000 & 0.946 & 0.973 & 1.000 & 0.743 & 0.872 & 1.000 & 0.833 & 0.917 \\
\hline 8 & 0.770 & 1.000 & 0.885 & 0.910 & 1.000 & 0.955 & 0.895 & 1.000 & 0.947 & 0.764 & 1.000 & 0.882 \\
\hline 9 & 0.500 & 1.000 & 0.750 & 0.599 & 1.000 & 0.799 & 0.656 & 1.000 & 0.828 & 0.552 & 1.000 & 0.776 \\
\hline 10 & 1.000 & 0.668 & 0.834 & 1.000 & 0.760 & 0.880 & 1.000 & 0.611 & 0.805 & 1.000 & 0.685 & 0.843 \\
\hline
\end{tabular}




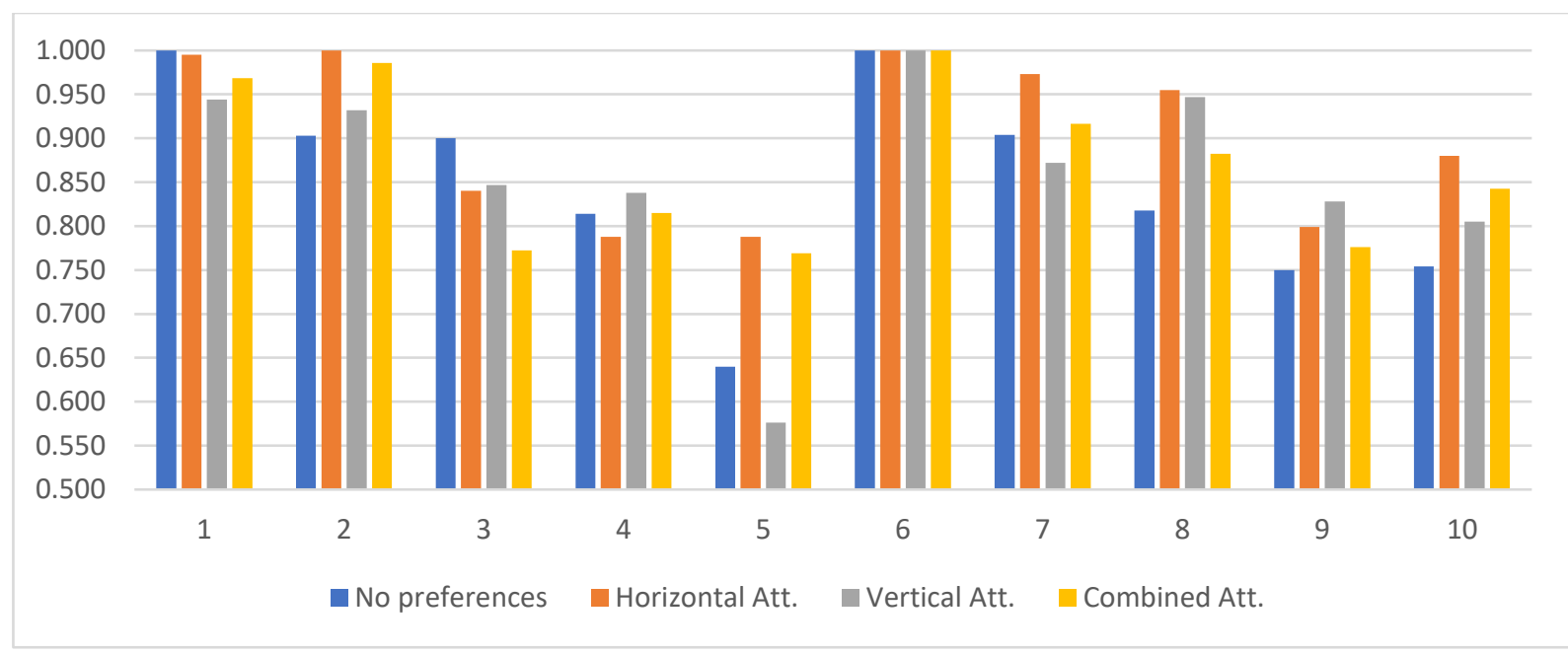

Figure 3: Distribution of chains' efficiency scores

Figures 4, 5, and 6 compare chains' efficiency score distributions and the distribution of weights representing DMsP in the cases of horizontal, vertical and combined attributions, respectively. It is obvious that efficiency scores vary from one chain to another, but it can be seen from the figures that these variations are not caused by the deviation of preference weights. Indeed, as shown in Table 6, the correlations between these weights and the efficiency scores in all the proposed cases are very low. This result is in line with the objective we set that is the incorporation of DMsP without affecting the DEA mechanism.

By analyzing the different results generated by the GAA in order to create a consistent measure of supply chains' efficiencies, we can deduce that the efficiency scores in the different proposed cases are not sensitive to the variation of weights, thus reflecting the preferences of DMs toward chains and their corresponding members.

This is an important advantage compared to traditional methods for incorporating preferences into DEA models, including weight restriction methods (Absolute weight restrictions, Assurance region of type I, Assurance region of type II, weight restrictions on virtual input and output; see Allen et al. (1997). These methods incorporate preferences by adding restrictions on the weights in the multiplier DEA models. Thus, there may be substantial changes to the mix of inputs and outputs of a given supply chain, and deteriorations in some observed input or output levels.

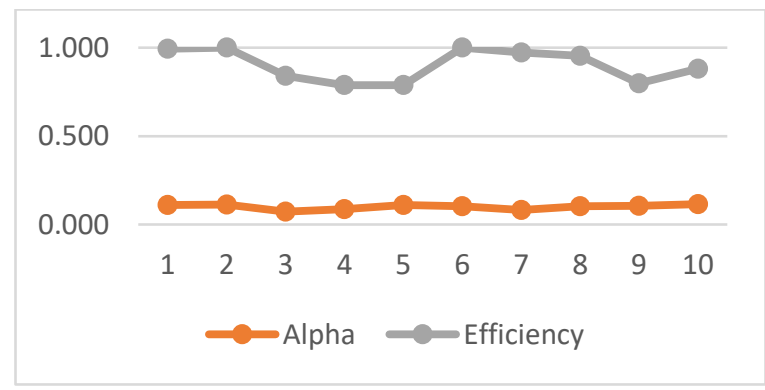

Figure 4: Efficiency dist. Vs weight dist. (horizontal attribution) 


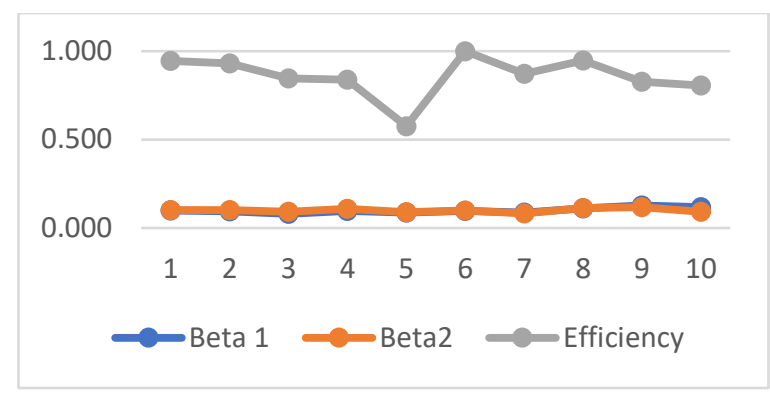

Figure 5: Efficiency dist. Vs weight dist. (vertical attribution)

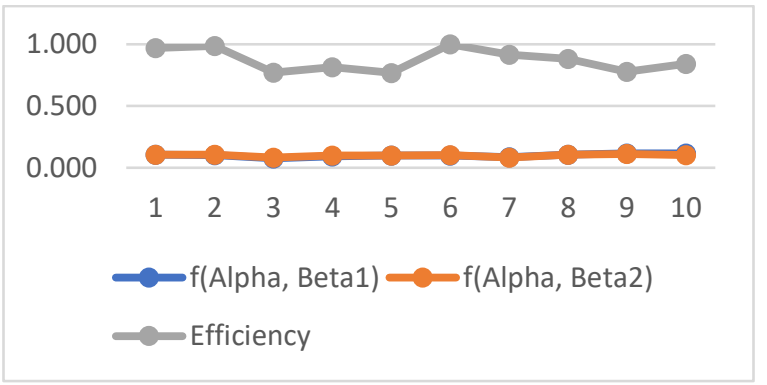

Figure 6: Efficiency dist. Vs weight dist. (combined attribution)

Table 6: Correlation between weights and efficiency scores

\begin{tabular}{lc}
\hline Correlation between & $R$ \\
\hline$E_{S C_{k}}^{H}$ and $a_{k}$ & 0.205 \\
$E_{S C_{k}}^{V}$ and $\beta_{d S_{k}}$ & 0.132 \\
$E_{S C_{k}}^{V}$ and $\beta_{d M_{k}}$ & 0.340 \\
$E_{S C_{k}}^{C}$ and $P_{k}\left(\alpha_{d k}, \beta_{d S_{k}}\right)$ & 0.035 \\
$E_{S C_{k}}^{C}$ and $P_{k}\left(\alpha_{d k}, \beta_{d M_{k}}\right)$ & 0.131 \\
\hline
\end{tabular}

At the managerial level, the GAA approach has the advantage of being more flexible. In fact, DMs may express their judgments on any given supply chain based on a priori information and knowledge, such as the relative importance of the supply chain related to its location, its historical significance, its strategic weight, its capital gain.... Moreover, each member belonging to a given supply chain may specialize in a specific input-output mix not directly comparable with that of another member. Thus, in order to prevent the inputs or outputs from being overemphasized or ignored in the assessment, a discrimination between supply chains and/or members was applied by considering such relevant a priori information so that DMsP will be reflected in the outcomes of the assessments.

In addition, at the modeling level, the proposed GAA also has another advantage. Indeed, without having to resort to data transformations or to other methods beside the DEA, the incorporation of DMsP into models is more direct. Finally, some extra empirical benefits may be added to these advantages. (1) Controlling the sample size: By choosing not to reveal his preferences, a given DM would be able to eliminate the first or the second member by assigning a zero weight to it. In the case of horizontal attribution of preferences, he could eliminate a whole chain by assigning a zero weight. In the case of the vertical attribution of preferences, he could eliminate a whole chain by assigning two zero weights, one to each member. (2)Assisting in sensitivity analysis: By comparing the results yielded from the different manners proposed to consider the attribution of preferences (absence, horizontal, vertical and combined), it will be easy to detect and analyze plausible variations of efficiencies generated by each considered case. 


\section{Conclusion}

The main objective of this research is to contribute to the improvement of DEA procedures related to measuring the technical efficiency of supply chains by taking into account a very important factor, which is the preferences of DMs. A novel approach to consider the attribution of these preferences was suggested, and new DEA models were developed. The importance of this approach is derived from the fact that DMs could express their judgments on the supply chains and corresponding members under assessment.

The benefits of this approach have been highlighted using an illustrative example. It shows more flexibility and a relatively easier use compared to existing approaches that deal with the same line of research. In particular, it keeps inherent the DEA philosophy that retains the weights on inputs and outputs as variables.

However, there are several limitations of our study. One limitation is the considered configuration of supply chains was limited to simple linear two-member chains. This work is likely to be generalized in the future by trying to adopt more complex supply chains. A second limitation is that weights used to reflect DMsP are subjective. Further research is required in order to apply the proposed approach to a real example by using real weights.

\section{Acknowledgments}

The authors gratefully acknowledge the approval and the support of this research study by the grant no. 7056-SAR2017-1-7-F from the Deanship of Scientific Research at Northern Border University, Arar, K.S.A.

\section{References}

1. Abdelfattah, W., Rebai, A. (2016). Measurement of dyadic supply chains efficiency under new assumptions using DEA models. Journal of Applied Science 16:445-453.

2. Alcaide-López-de-Pablo, D., Dios-Palomares, R., Prieto, Á. M. (2014). A new multicriteria approach for the analysis of efficiency in the Spanish olive oil sector by modelling decision maker preferences. European Journal of Operational Research 234:241-252.

3. Allen R., Athanassopoulos, A., Dyson, R. G., Thanassoulis, E. (1997). Weights restrictions and value judgements in data envelopment analysis: evolution, development and future directions. Annals of Operations Research 73:13-34.

4. Arabshahi, H., Fazlollahtabar, H., Maboudi, L. (2020). Efficiency Evaluation of Supply Chain Network Using a Framework Based on DEA and Seller-Buyer Structure. Asia-Pacific Journal of Operational Research DOI: $10.1142 / \mathrm{S} 0217595920500293$.

5. Balfaqih H., Nopiah, Z. M., Saibani, N., Al-Nory, M. T. (2016). Review of supply chain performance measurement systems: 1998-2015. Computers in Industry 82:135-150.

6. Chen, Y., Cook, W. D., Li, N., Zhu, J. (2009). Additive efficiency decomposition in two-stage DEA. European Journal of Operational Research 196:1170-1176.

7. Chen, Y., Liang, L., Yang, F. (2006). A DEA game model approach to supply chain efficiency. Annals of Operations Research 145:5-13.

8. Contreras, I. (2011). A DEA-inspired procedure for the aggregation of preferences. Expert Systems with Applications 38:564-570.

9. Cook, W., Liang, L., Zhu, J. (2010). Measuring performance of two-stage network structures by DEA: a review and future perspective. Omega 38:423-430.

10. Cook, W. D., Seiford, L. M. (2009). Data envelopment analysis (DEA) - thirty years on. European Journal of Operational Research 192:1-17.

11. Emrouznejad, A., Parker, B.R., Tavares, G. (2008). Evaluation of research in efficiency and productivity: a survey and analysis of the first 30 years of scholarly literature in DEA. Socio-Economic Planning Sciences 42:151-157.

12. Emrouznejad, A., Yang, G. L. (2018). A survey and analysis of the first 40 years of scholarly literature in DEA: 1978-2016. Socio-Economic Planning Sciences 61 (1): 4-8.

13. Kao, C., Hwang, S.N. (2008). Efficiency decomposition in two-stage data envelopment analysis: an application to non-life insurance companies in Taiwan. Eurpean Journal of Operational Research 185:418-429.

14. Kaviani, M. A., Abbasi, M. (2014). Analyzing the operations strategies of manufacturing firms using a hybrid grey DEA approach - a case of fars cement companies in Iran. International Journal of Supply Operations Management 1:371-391. 
15. Liang, L., Yang, F., Cook, W. D., Zhu J. (2006). DEA models for supply chain efficiency evaluation. Annals of Operations Research 145:35-49.

16. Maghbouli, M., Amirteimoori, A., Kordrostami, S. (2014). Two-stage network structures with undesirable outputs: a DEA based approach. Measurement 48:109-118.

17. Matin, R. K., Azizi, R. (2015). A unified network-DEA model for performance measurement of production systems. Measurement 60:186-193.

18. Parkan, C., Wang J. (2007). Gauging the performance of a supply chain. International Journal of Productivity and Quality Management (IJPQM) 2:141-176.

19. Saen, R. (2010). Restricting weights in supplier selection decisions in the presence of dual-role factors. Applied Mathematical Modelling 34:2820-2830.

20. Seiford, L. M., Zhu, J. (1999). Profitability and marketability of the top 55 U.S. Commercial banks. Management Science 45:1270-1288.

21. Tavana, M., Kaviani, M. A., Di Caprio, D., Rahpeyma, B. (2016). A two-stage data envelopment analysis model for measuring performance in three-level supply chains. Measurement 78:322-333.

22. Wang, Y. M., Chin, K.S. (2010). Some alternative DEA models for two-stage process. Expert Systems with Applications 37:8799-8808.

23. Wang, C. N., Nguyen, T.V., Duong, H.D., Do, T.H. (2018). A Hybrid Fuzzy Analytic Network Process (FANP) and Data Envelopment Analysis (DEA) Approach for Supplier Evaluation and Selection in the Rice Supply Chain. Symmetry 10:221-236.

24. Wong, W. P. (2009). Performance evaluation of supply chain in stochastic environment: using a simulation based DEA framework. International Journal of Business Performance and Supply Chain Modelling (IJBPSCM) 1:203228.

25. Xu, J., Li, B., Wu, D. (2009). Rough data envelopment analysis and its application to supply chain performance evaluation. International Journal of Production Economics 122:628-638.

26. Yang, F., Wu, D., Liang, L., Bi, G., Wu, D. D. (2009). Supply chain DEA: production possibility set and performance evaluation model. Annals of Operations Research 185:195-211.

27. Zhao, X., Sun, L. (2008). A preference restraint DEA approach for supplier selection. In: 2008 4th International conference on wireless communications, networking and mobile computing. IEEE, Dalian, China, pp 1-4.

28. Zhai, D., Shang, J., Yang, F., Ang, S. (2019). Measuring energy supply chains' efficiency with emission trading: A two-stage frontier-shift data envelopment analysis. J. Clean. Prod. 210:1462-1474.

29. Zhu, J. (2003). Quantitative models for performance evaluation and benchmarking: data envelopment analysis with spreadsheets. Kluwer Academic Publishers, Boston 